\title{
LUT
}

Lappeenranta

University of Technology

\section{Simulation Decomposition: New Approach For Better Simulation Analysis Of Multi-Variable Investment Projects}

Kozlova Mariia, Collan Mikael, Luukka Pasi

\author{
This is an Pre-print \\ version of an article \\ published by SIGEF \\ in Fuzzy Economic Review
}

DOI: $10.25102 / f e r .2016 .02 .01$

Copyright of the original publication: () SIGEF 2017

Please cite the publication as follows:

Citation. E.g. Kozlova, M., Collan, M,. Luukka, P. (2017). Simulation Decomposition: New Approach For Better Simulation Analysis Of Multi-Variable Investment Projects. Fuzzy Economic Journal, Vol 21, Issue 2. p. 3-18. DOI:10.25102/fer.2016.02.01

This is a parallel published version of an original article. This version can differ from the original published article. 
SIMULATION DECOMPOSITION: NEW APPROACH FOR BETTER

\section{SIMULATION ANALYSIS OF MULTI-VARIABLE INVESTMENT PROJECTS}

Mariia Kozlova, ${ }^{a}$ Mikael Collan, ${ }^{b}$ and Pasi Luukka ${ }^{c}$

a,b,c School of Business and Management, Lappeenranta University of Technology

Skinnarilankatu 34, 53850 Lappeenranta, Finland

${ }^{a}$ corresponding author, mariia.kozlova@lut.fi, +358 504636168 


\title{
SIMULATION DECOMPOSITION: NEW APPROACH FOR BETTER
}

\section{SIMULATION ANALYSIS OF MULTI-VARIABLE INVESTMENT PROJECTS}

\begin{abstract}
This paper presents a new method to enhance simulation-based analysis of complex investments that contain multi-variable uncertainty. The method is called "simulation decomposition". Typically the result of simulation-based investment analysis is in the form of histogram distributions - here we propose a method for first classifying the possible outcomes of selected uncertain variables into states and then using combinations of the created states in the decomposition of the simulated distribution into a number of sub-distributions. The sub-distributions that can be matched to state-combinations of the variables contain relevant actionable information that helps managers in decision-making with regards to the studied investments.
\end{abstract}

A numerical illustration of a renewable energy investment is used to demonstrate the usability, the enhanced analytical power, and the intuitively understandable benefits that can be reached by using the simulation decomposition method. The proposed method is generally usable and can be utilized independent of the investment context.

Keywords: Corporate Finance and Governance, Capital Budgeting, Simulation Modeling, Renewable Resources and Conservation 


\section{INTRODUCTION}

In this paper we are interested in exploring how typical simulation based investment analysis can be enhanced to offer better managerial decision-support in terms of providing better actionable information about threshold values for identified important-to-the-investment variables that affect investment profitability. Our focus is on analysis that is performed before the investment decision. The decision support before investment is "actionable", because it is before the investment that decision managers are often in a position that allows them to still plan and steer investments towards the most profitable configurations, and by their actions ensure that critical to profitability issues are properly accounted for.

What we propose is a new approach that we call "simulation decomposition". The method is based on setting artificial (expert chosen) thresholds to divide the possible value distributions of the most important uncertain variables of an analyzed investment. Typically in Monte Carlo simulation these distributions are from where the simulator draws random variable values. After having "decomposed" each variable's uncertainty, or "range", into sub-ranges, the combinations of these sub-ranges are listed. When the simulation is run, the results are registered separately for each combination, in addition to the overall simulation results. This allows for constructing a separate distribution of outcomes for each combination that is a sub-distribution of the overall simulation result. By studying the subdistributions managers can infer important information about the profitability-critical threshold values for each variable that will help them plan their actions with regards to managing the investment better.

Modern investment decision-making is most often an exercise that involves comparing the value of an upfront investment cost and a stream of uncertain future cash-flows that is expected to result from the prospective investment. In practice, the methods "used for the job" are the "classical" capital budgeting methods, such as the net present value (NPV) method, the pay-back method, and the internal rate of return method (IRR) that are often used

\section{Abbreviations}

CapEx - Capital expenditures

$\mathrm{CPI}$ - Consumer price index

IRR - Internal rate of return

MCS - Monte Carlo simulation

MW - Megawatt

NPV - Net present value

PV - Photo-voltaic

$\mathrm{RE}$ - Renewable energy

ROV - Real option value 
together with complementary sensitivity, scenario, and simulation analysis methods (Block, 2007; Graham \& Harvey, 2001; Ryan \& Ryan, 2002). Real option analysis (Amram \& Kulatilaka, 1998; Trigeorgis, 1995) is among the latest additions into the investment analysis toolkit of managers and has been gaining a foothold in academic research, as well as, a following in the industry. The benefit of real option analysis over the classical methods is that it is able to capture the value of managerial flexibility that is to be found within investments, and when investments are considered as a whole. Often a mixture of different investment analysis techniques is used simultaneously in hopes of gaining a better holistic picture of the situation surrounding the investment and in order to comprehensively treat the risks involved.

Simulation and more specifically Monte Carlo simulation (commonly attributed to Stanislaw Ulam), is a technique that has been used in asset valuation since the late 1970's, e.g., (Boyle, 1977), and in investment analysis of real investments for more than two decades. In connection with the classical profitability analysis methods simulation has been used, e.g., in enhancing scenario analysis for complementing the investment analysis (Sheel, 1995). Simulation has also been used together with dynamic system models in investment analysis and profitability evaluation of, e.g., mining and oil investments (Johnson, Taylor, \& Ford, 2006; O'Regan \& Moles, 2001; O'Regan \& Moles, 2006; Sontamino \& Drebenstedt, 2014; Tan, Anderson, Dyer, \& Parker, 2010), and to offer a better understanding of how uncertain variables affect profitability of energy investments (Bastian-Pinto, Brandão, \& Alves, 2010; Boomsma, Meade, \& Fleten, 2012; Kozlova, Collan, \& Luukka, 2015; Monjas-Barroso \& Balibrea-Iniesta, 2013; Reuter, Fuss, Szolgayová, \& Obersteiner, 2012; Vithayasrichareon \& MacGill, 2012; Yang, Nguyen, De T'Serclaes, \& Buchner, 2010). Simulation is also an underlying technique in many modern real option valuation methods such as the Datar-Mathews method (Datar \& Mathews, 2004; Mathews, Datar, \& Johnson, 2007) and the Matching Method (Jaimungal \& Lawryshyn, 2015). Typically what is achieved as an end result from a simulation based profitability analysis is a histogram (distribution) of project profitability, a NPV distribution. Simulation is also used in many fields of engineering as a standard multi-purpose tool; these other purposes are however left outside the scope of this paper, but we suggest the interested reader to see, e.g., (García-Redondo, López-Vallejo, Ituero, \& Barrio, 2012; Rane et al., 2014). The proposed method may be useful in providing new insight in such simulationbased analysis as e.g. (Nembhard, Shi, \& Aktan, 2003; Ridlehoover, 2004; Savolainen, Collan, \& Luukka, 2016).

Although the Monte Carlo simulation technique has been applied to many different problems throughout the years, 
the simulation method itself and the way the simulation results are analyzed has remained relatively unchanged. Here we propose an enhancement to the simulation procedure and as a consequence to the analysis of the received results that enhances the explanatory power of the results in terms of increased understanding of the cause-effect relationship between the variables and the simulation results.

The proposed new approach is illustrated numerically with a case that is based on a renewable energy investment into Russia that may enjoy the support offered by the Russian renewable energy incentive mechanism (Government of Russian Federation, 2013a; Government of Russian Federation, 2013b). The case is especially suitable for the task, as the complex incentive mechanism "naturally" creates what can be called artificial thresholds for the variables that are important to the profitability of the investment (Boute, 2012; International Finance Corporation, 2011; Kozlova, 2015; Vasileva, Viljainen, Sulamaa, \& Kuleshov, 2015).

The remainder of the paper is structured as follows: the following section presents the proposed simulation approach in more detail, then a numerical case illustration based on a renewable energy investment in Russia is presented to show real-world use of the approach, and finally conclusions are drawn and some future research directions are identified.

\section{THE NEW SIMULATION DECOMPOSITION APPROACH}

It needs to be observed first that we expect that a simulation model has been built and on the model one can run Monte Carlo simulation. As this is in place we directly present how the simulation procedure is to be changed according to the simulation decomposition approach. In order to do this the simulation decomposition process is presented as a five step algorithm:

Step 1: Identify key variables $\tilde{V}_{i}$ and the possible range of the values that each identified key variable can take.

The first step is to start from identifying key variables, where important to the project profitability uncertainty exists. These can be identified by consulting the managers inside the organization involved in the planning of the investment project, or by simply performing typical sensitivity analysis to find out which variables are the most important. These variables are "the same variables" that would "normally" be identified, when a classical Monte Carlo simulation model 
is built, and for which values are simulated. In the case example used in this paper five uncertain key variables were identified. After identification, the range of values that each identified key variable may have is estimated. The determination of the ranges can be done by asking managers to identify them by, for example, giving minimum and maximum possible values for each variable, or by deriving the range from historical data. It is worth mentioning that only this first step belongs also to the procedure used in the classical Monte Carlo simulation (Mooney, 1997), while the rest form a unique basis for the decomposition approach.

Step 2: Identify key variables that the organization can affect and relevant states $\tilde{A}_{i j}$ for these.

The second step in the simulation decomposition approach is to identify two, or more, "managerially relevant" states $\tilde{A}_{i j}$ for the identified key variables that the organization undertaking the project can affect. This part of the process is specific to each problem under analysis, but the point is that the identified states point to different outcomes in terms of project success, or organizational achievement in terms of the variable in question. For instance, in the example used in this paper, states of "failure" or "success" can be identified with regards to reaching a level of production that "earns" the project a subsidy. Typically, when there are no naturally "given" or derivable states they can be managerially determined, based perhaps on issues such as, "certainly reachable" variable values, "usual" or "normal" values of a variable, "good" or "sufficient" variable values, and so forth. The number of identified states may very well be only two, for example, "good" and "bad" - this is not a problem in terms of how the simulation decomposition approach can be used. In the case example used in this paper one identified key variable has three states and two key variables have two states. States are not identified for variables whose values the organization cannot affect, such as market prices.

\section{Step3: Identify suitable boundaries $\tilde{B}_{i j}$ for each state $\tilde{A}_{i j}$.}

The third step of the decomposition analysis is to identify the boundaries $\tilde{B}_{i j}$ between the identified states $\tilde{A}_{i j}$. For binary variables the boundary is naturally between the two states $\widetilde{B}_{i j}=\{0,1\}$, if one is dealing with a real valued variable, boundaries of the variable are specified by using a threshold value $\tilde{t}_{i}$, e.g., $\tilde{B}_{i 1}=\left[0, \tilde{t}_{i}\right] \tilde{B}_{i 2}=\left(\tilde{t}_{i}, 100\right]$, where the two identified states of the variable $\tilde{V}_{i}$ for which the range of possible outcomes is $[0,100]$ (for example \%) are separated by the threshold value $\tilde{t}_{i}$. Similarly $\left(N_{i}-1\right)$ threshold values are identified for $i^{\text {th }}$ variable with more than two states. For the purposes of this research we focus on using crisp threshold values, uncertainty can be introduced in the threshold values i.e. by using fuzzy sets, but we leave discussion about fuzzy threshold values as 
a matter of further research and outside the scope of this research.

Step 4: Form groups $\tilde{G}_{k}$ of combinations to specify the to-be-decomposed parts of the simulation.

The fourth step is to form relevant groups $\tilde{G}_{k}$ of possible outcomes to investigate by selecting a possible state $\tilde{A}_{i j}$ from each variable $\tilde{V}_{i}$. The resulting list of groups represents all possible combinations of the states of the key variables. The total number of groups is, therefore, a product of a number of the states of the key variables, $\prod_{i=1}^{n} N_{i}$, where $N_{i}$ denotes the number of states in $i^{\text {th }}$ variable. For example, having two key variables $\tilde{V}_{1}$ and $\tilde{V}_{2}$ with two states identified for each variable, the total number of groups is four and is comprised of $\widetilde{G}_{1}=\left\{\tilde{A}_{11}, \tilde{A}_{21}\right\}, \tilde{G}_{2}=$ $\left\{\tilde{A}_{11}, \tilde{A}_{22}\right\}, \tilde{G}_{3}=\left\{\tilde{A}_{12}, \tilde{A}_{21}\right\}, \tilde{G}_{4}=\left\{\tilde{A}_{12}, \tilde{A}_{22}\right\}$ (where first index stands for the variable index and the second one for the state). Each group corresponds to a piece of the total simulation, what is essentially done is a specification of the to-be-decomposed parts of the simulated (NPV) distribution for the project.

Step 5: Run the simulation model, while assigning the result of each simulation round not only to the distribution of the "full" simulation, but also to the specified decomposed group $\tilde{G}_{k}$ or sub-distribution of the simulation.

The fifth step is to run the simulation model $n$ times and to track the resulting NPV value of each simulation round and to store it not only as a part of the "full" NPV distribution from the project, but also as a part of a specifically identified "decomposed" NPV sub-distribution NPV $\left(\right.$ here NPV $V_{k}$ denotes NPV of group $\tilde{G}_{k}$ ). The identification of the correct sub-distribution for each round is done by mapping randomly drawn values from each variable $\tilde{V}_{i}$ to its corresponding state $\tilde{A}_{i j}$ and finding a correct group $\tilde{G}_{k}$ by matching states $\tilde{A}_{i j}$ with corresponding group $\tilde{G}_{k}$. In practice this can be established, for example, by using lookup tables, or by running an if-loop over the variables to find, which particular states are valid for a given round of the simulation run. This way we end up with having $\prod_{i=1}^{n} N_{i}$ sub-distributions.

For all steps $1-5, i=1, \cdots, n, j=1, \cdots, N_{i}, k=1, \cdots, \prod_{i=1}^{n} N_{i}, n$ is number of variables, and $N_{i}$ is number of possible states in $i^{\text {th }}$ variable.

After this five-step approach is run, one is left with a typical simulated (NPV) distribution for the (whole) project and $\prod_{i=1}^{n} N_{i}$ sub-distributions, $\mathrm{NPV}$, each specified for a particular group $\tilde{G}_{k}$. These can then be used to help plan the 
managerial actions connected to the prospective investment and to understand better the drivers of the profitability of the investment. The simulation decomposition approach can be easily used on top of almost any existing simulation model. The method is also flexible in terms of the software environment used. The main requirement for successfully implementing the method on top of a suitable existing simulation model is constructing a block that matches the (NPV) result of each simulation round with the correct sub-distribution.

\section{NUMERICAL CASE ILLUSTRATION}

The case used to illustrate the method is an analysis of a renewable energy (RE) investment into a $10 \mathrm{MW}$ solar photo-voltaic (PV) power plant in Russia. The revenues from this investment comprise of electricity sales in the Russian wholesale energy market and of subsidy cash-flows from a long-term capacity delivery contract. The latter is calculated based on the rules laid-out in the Russian law governing the incentive mechanism for RE investments that has the target of providing a certain level of return on investment. The mechanism considers changing market conditions and project-specific characteristics, for details see (Government of Russian Federation, 2013a; Government of Russian Federation, 2013b). The Russian mechanism is different from the typically used RE incentive schemes in use, e.g., in Central Europe. For more on the Russia RE support mechanisms see (Boute, 2012; International Finance Corporation, 2011; Kozlova, 2015; Vasileva et al., 2015).

The investment analysis model for the solar power plant investment is a simple system dynamic model that combines a typical discounted cash-flow calculation with a model of the Russian RE investment incentive mechanism. Block diagram of the model used is presented in Figure 1, where input variables are located on the left-hand side of the model and outputs are visible on the right. The two largest blocks represent the main computational parts of the model, where the effects of the incentive mechanism are determined for each simulation run and the resulting project NPV are calculated. A more detailed description of the case and the assumptions behind the model used can be found in (Kozlova, 2015). 


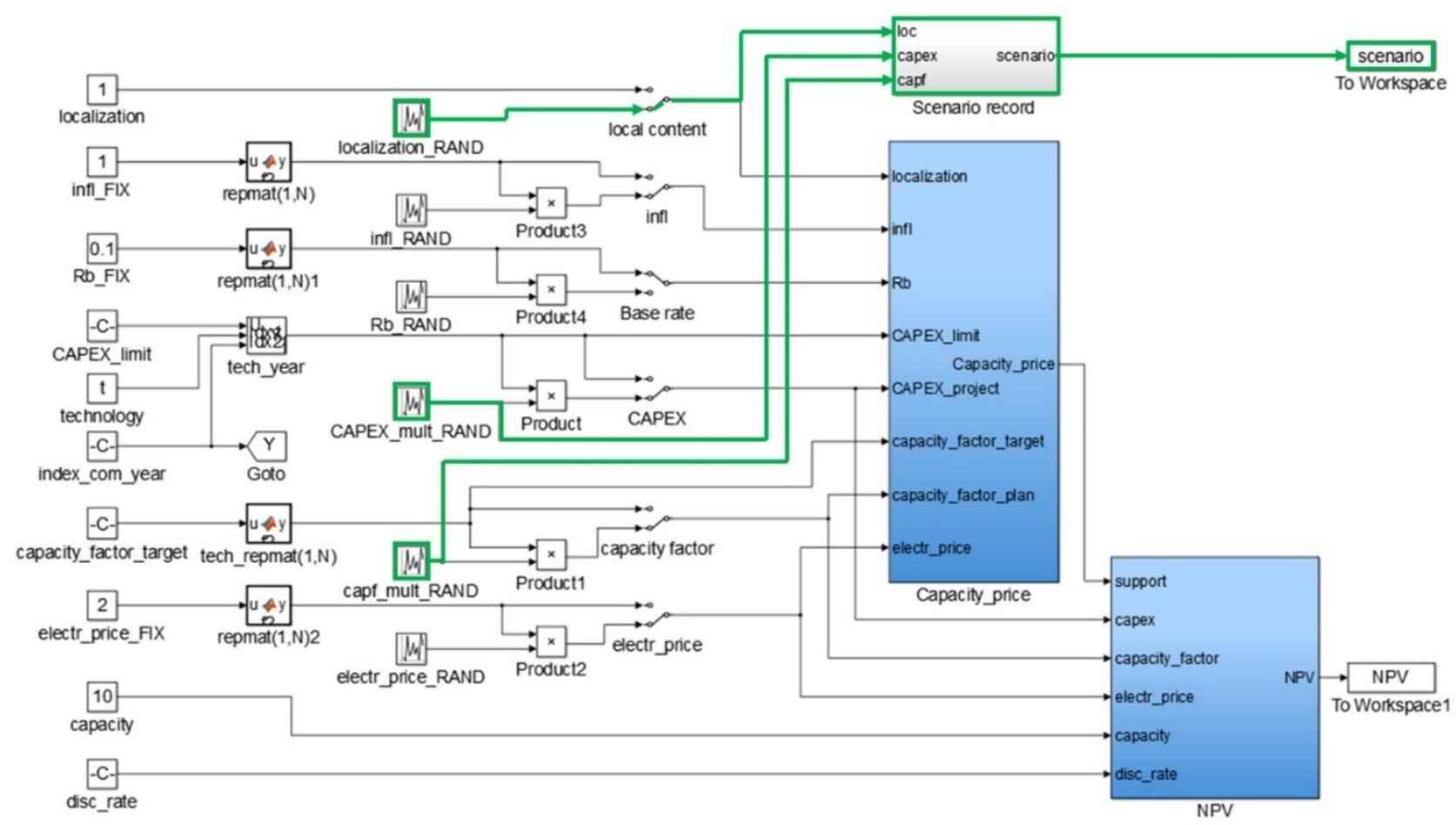

Figure 1. Block-diagram of the used model

The model has been built in Matlab Simulink and the simulations are run ten thousand times (rounds). Next we go through the simulation decomposition procedure in the order of the algorithm presented above.

\subsection{SIMULATION DECOMPOSITION FOR THE CASE IN AN ALGORITHMIC FORM}

First step is the identification of the (uncertain) key variables and the possible range of values each key variable can take. The identified key variables are five:

$$
\tilde{V}=\left\{\tilde{V}_{1}, \tilde{V}_{2}, \tilde{V}_{3}, \tilde{V}_{4}, \tilde{V}_{5}\right\}=\{\text { Capacity factor, Localization, CapEx level,CPI,Electricity price }\}
$$

and the possible range of each key variable's value is presented in Table 1. For the purposes of this paper the ranges from within which the random draws are made are modeled as uniform distributions (probability is the same for drawing any value from within the range). 
Table 1. Uncertain key variables for the RE investment

\begin{tabular}{|l|l|l|}
\hline \multirow{2}{*}{\multicolumn{1}{|c|}{ Key variable }} & \multicolumn{2}{c|}{ Possible range of values } \\
\cline { 2 - 3 } & \multicolumn{1}{|c|}{ Minimum } & \multicolumn{1}{c|}{ Maximum } \\
\hline Electricity price, rub./MWh & 1000 & 3000 \\
\hline Consumer price index CPI (inflation) & 1.00 & 1.70 \\
\hline Capital expenses (CapEx) level & $80 \%$ & $150 \%$ \\
\hline Capacity factor (percent of target) & $30 \%$ & $120 \%$ \\
\hline Localization requirement & Failed (0) & Fulfilled (1) \\
\hline
\end{tabular}

The second step is to identify the key variables the organization can affect and to identify relevant states for these variables. The first two factors in Table 1, electricity price and inflation, represent external or "market" factors that are outside the influence of the investor, whereas the remaining three variables represent project-internal factors that can be managed to some extent by project managers: \{ Capacity factor, Localization, CapEx level $\}$. The relevant states to be studied for each of these three key variables are:

Capacity factor $=\left\{\tilde{A}_{11}, \tilde{A}_{12}, \tilde{A}_{13}\right\}=\{$ Low, Medium, High $\}$

Localization $=\left\{\tilde{A}_{21}, \tilde{A}_{22}\right\}=\{$ Failed, Fulfilled $\}$

CapEx level $=\left\{\tilde{A}_{31}, \tilde{A}_{32}\right\}=\{$ within the limit, over the limit $\}$

Thus we have one three-state variable and two binary variables. The identified "states" directly and realistically reflect the features of the Russian RE investment incentive mechanism. The level of the capital expenditures, the level of annual electricity production (capacity factor), and fulfilling a requirement for local production in the investment (localization) affect the amount of support that the investment can receive in the form of RE capacity payments. For example, failing the localization requirement will cut the received RE capacity payments to roughly a half of the 
maximum. Details of the Russian RE incentive mechanism and the effect of the three variables can be found, e.g., in (Government of Russian Federation, 2013a; Government of Russian Federation, 2013b; Kozlova \& Collan, 2016; Kozlova, 2015).

The third step is to identify what was called "suitable boundaries" for the identified states. This means setting, in this case numerical, thresholds $\tilde{t}_{i j}$ that separate the different states, while observing the bounds of each state. Here these thresholds are given by the underlying Russian RE incentive mechanism: for "Capacity factor" we have two threshold values $\tilde{t}_{11}=50 \%$ and $\tilde{t}_{12}=75 \%$ and the boundaries of the three resulting states become $\tilde{B}_{11}=$ $[30 \%, 50 \%], \tilde{B}_{12}=(50 \%, 75 \%]$, and $\tilde{B}_{13}=[75 \%, 120 \%]$, where the minimum and the maximum we get from the range of possible values, see Table 1 . Similarly for the binary variable "Localization" we have $\tilde{B}_{21}=0$ and $\tilde{B}_{22}=1$ and for "CapEx level" $\widetilde{B}_{31}=[80 \%, 100 \%]$ and $\widetilde{B}_{32}=(100 \%, 150 \%]$.

The fourth step is to form groups $\tilde{G}_{k}$ from a list of "combinations of states" between different variables, these combinations will be the to-be-decomposed parts of the simulation. The combinations of states can also be called "scenarios" as denoted in block-diagram in Figure 1. Simulation results for each round are matched to these scenarios. In this particular case we get 12 scenarios that are listed in Table 2.

Table 2. List of different scenarios with states of the variables identified and boundaries indicated

\begin{tabular}{|c|l|l|l|}
\hline Scenario, $\tilde{G}$ & Capacity factor (percent of target) & Localization & CapEx level \\
\hline 1 & High $(75 \%, 120 \%]$ & Fulfilled & Within the limit $[80 \%, 100 \%]$ \\
\hline 2 & High $(75 \%, 120 \%]$ & Fulfilled & Over the limit $(100 \%, 150 \%]$ \\
\hline 3 & High $(75 \%, 120 \%]$ & Failed & Within the limit $[80 \%, 100 \%]$ \\
\hline 4 & High $(75 \%, 120 \%]$ & Failed & Over the limit $(100 \%, 150 \%]$ \\
\hline 5 & Medium $(50 \%, 75 \%]$ & Fulfilled & Within the limit $[80 \%, 100 \%]$ \\
\hline 6 & Medium $(50 \%, 75 \%]$ & Fulfilled & Over the limit $(100 \%, 150 \%]$ \\
\hline 7 & Medium $(50 \%, 75 \%]$ & Failed & Within the limit $[80 \%, 100 \%]$ \\
\hline 8 & Medium $(50 \%, 75 \%]$ & Failed & Over the limit $(100 \%, 150 \%]$ \\
\hline
\end{tabular}




\begin{tabular}{|c|l|l|l|}
\hline Scenario, $\tilde{G}$ & Capacity factor (percent of target) & Localization & CapEx level \\
\hline 9 & Low $(30 \%, 50 \%]$ & Fulfilled & Within the limit $[80 \%, 100 \%]$ \\
\hline 10 & Low $(30 \%, 50 \%]$ & Fulfilled & Over the limit $(100 \%, 150 \%]$ \\
\hline 11 & Low $(30 \%, 50 \%]$ & Failed & Within the limit $[80 \%, 100 \%]$ \\
\hline 12 & Low $(30 \%, 50 \%]$ & Failed & Over the limit $(100 \%, 150 \%]$ \\
\hline
\end{tabular}

The fifth step is to run the simulation model, while matching the result of each simulation round (the NPV of the RE investment) not only to the results of the "full" simulation, but also specifically to each of the twelve scenarios. Matching is based on the correspondence of the randomly drawn values for the three variables to the particular states identified by the boundaries of the states. For example, if randomly drawn capacity factor is " $79 \%$ " that matches the state "high", randomly drawn localization "failed", and randomly drawn CapEx level "105\%" that matches the state "over the limit", the NPV from this simulation round would be attributed to scenario number four in Table 2. This process will finally create twelve sub-distributions, one for each scenario and the result will be a decomposition of the simulated distribution. The input-recording part of the used model is denoted with green arrows and block borders in Figure 1.

From the resulting NPV values for the project as a whole and for each scenario separately one can build histograms for the results. The parts must equal the whole. Typically, in a real-world analysis situation one would go about to, e.g., calculate descriptive statistics such as expected NPV, real option value, standard deviation of the NPV, et cetera. Next we look at the results from the numerical case illustration.

\subsection{SIMULATION RESULTS}

Simulation and simulation decomposition results are used to create histograms of the NPV for the whole project and for the twelve scenarios separately. The histogram for the whole project without decomposition is shown in Figure 2 on the left. What can be seen is that the form of the distribution is interesting. The shape of the distribution is caused by the effect of the Russian RE incentive policy. In Figure 2 on the right one can see the twelve scenarios' distributions inputted in the same picture and highlighted with different colors. Bars of different scenarios do not 
overlap in the histogram, but are "summed up" for each NPV value bin. What can be intuitively understood is "where" in the total distribution each scenario is present and what kind of initial values lead to which kinds of NPV results. Expected NPV and real option value (ROV in Figure 2) are indicated for both to highlight the fact that they are (and should be) the same, because both graphical presentations represent the same simulated NPV outcomes.
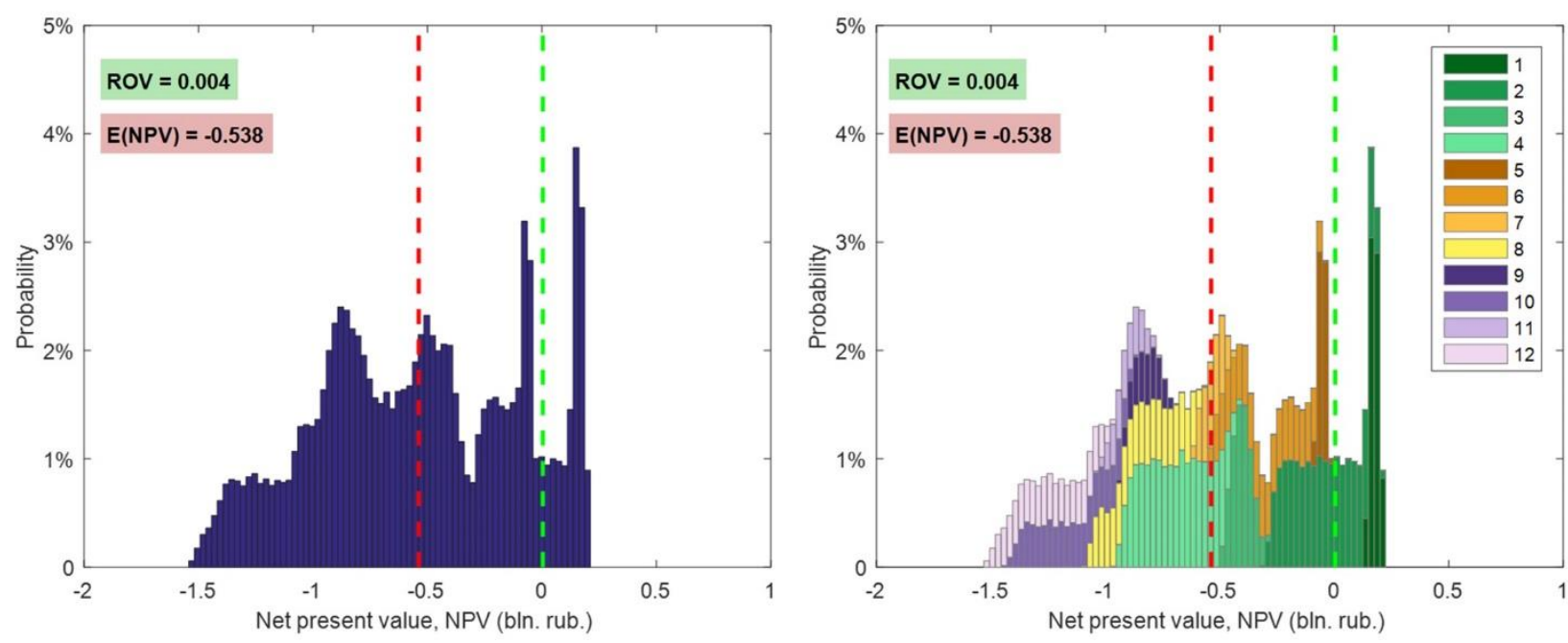

Figure 2. Simulated net present value (NPV) distributions. For legend key, see Table 2

Breaking down the overall simulation results into outcomes of different scenarios provides more information for decision-making. In particular, from the right graph of Figure 2 one can immediately see that the first scenario NPV's are entirely in the positive zone. This implies that an investor, who is able to manage the key variables to end up in the first scenario, can benefit from secure project profitability and independence from volatile market conditions. Another insight that can be easily obtained from observing the decomposed histogram is sensitivity of project profitability to the key variables. In the presented case, for instance, one can see the influence of electricity production performance by comparing the batches of green (1-4), yellow (5-8), and violet (9-12) scenarios. The gap between 1-2 and 3-4 scenarios (as well as between 5-6 and 7-8) appears due to failing localization requirement that implies severe effect on the investment profitability and, thus, requires particular management attention to ensure reaching this requirement. In contrast, capital cost increase is not such crucial (compare scenarios 1 and 2), and an investor can allow some overspending keeping the project profitable. All this information cannot be obtained from the conventional histogram alone. Thus, simulation decomposition reveals actionable information for planning an investment.

Table 3 presents a selection of calculated descriptive statistics from the results for the whole distribution and for each 
sub-distribution separately.

Table 3. Descriptive statistics

\begin{tabular}{|c|c|c|c|c|c|c|}
\hline & $\begin{array}{l}\text { Expected } \\
\text { NPV }\end{array}$ & ROV & $\begin{array}{l}\text { Standard } \\
\text { deviation }\end{array}$ & Minimum & Maximum & $\begin{array}{l}\text { Probability that a } \\
\text { simulation result } \\
\text { hit the scenario }\end{array}$ \\
\hline $\begin{array}{l}\text { Total } \\
\text { distribution }\end{array}$ & -538112 & 3888 & 444537 & -1543126 & 211925 & $100 \%$ \\
\hline Scenario 1 & 163426 & 163426 & 18153 & 123772 & 211925 & $7 \%$ \\
\hline Scenario 2 & -58143 & 22629 & 133644 & -325310 & 205211 & $18 \%$ \\
\hline Scenario 3 & -414466 & 0 & 42643 & -519048 & -284235 & $7 \%$ \\
\hline Scenario 4 & -701176 & 0 & 135105 & -971933 & -404229 & $18 \%$ \\
\hline Scenario 5 & -65746 & 0 & 13302 & -110960 & -30473 & $4 \%$ \\
\hline Scenario 6 & -308654 & 0 & 135064 & -577623 & -56636 & $10 \%$ \\
\hline Scenario 7 & -544211 & 0 & 40795 & -635715 & -439060 & $4 \%$ \\
\hline Scenario 8 & -842300 & 0 & 134252 & -1105438 & -565459 & $10 \%$ \\
\hline Scenario 9 & -834610 & 0 & 54708 & -966813 & -689509 & $3 \%$ \\
\hline Scenario 10 & -1164512 & 0 & 143629 & -1453132 & -867893 & $8 \%$ \\
\hline Scenario 11 & -916016 & 0 & 58886 & -1056890 & -766296 & $3 \%$ \\
\hline Scenario 12 & -1254252 & 0 & 143134 & -1543126 & -952481 & $8 \%$ \\
\hline
\end{tabular}

For this investment the only sub-distribution that lies entirely in the "positive zone" is that of the first scenario. Notice that also a part of the second scenario distribution is positive. In this case the difference is caused by the CapEx level (see Table 2) and further study shows that to be positive the project should fulfill the localization requirement, keep high average capacity factor, and make sure that the CapEx level is not higher than $120 \%$ of the limit. Figure 3 shows histograms of each sub-distribution separately with Expected NPV and ROV values. 

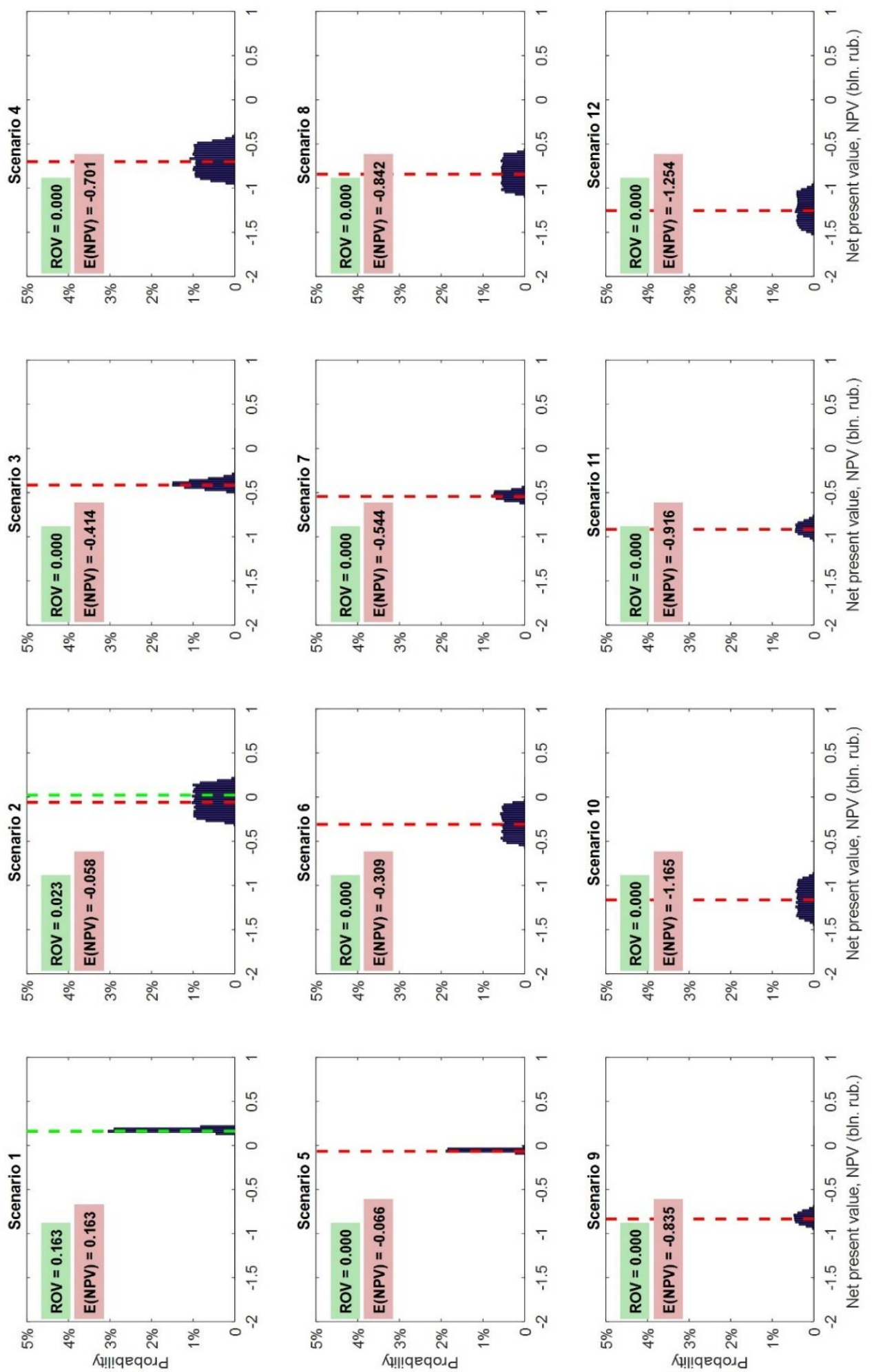

Figure 3. Histograms of each sub-distribution with expected net present values E(NPV) and real option values (ROV)

We also ran a two-sample Kolmogorov-Smirnov test of the resulting sub-distributions. This was done to make sure 
that our managerial decisions for different states and relevant scenarios was done in a way that was meaningful for the decision making process. This test confirmed that the samples are not drawn from same distribution when comparing the scenarios and further confirming our scenario selection (see Appendix I for details).

The decision-making implications for this case can be shortly summarized by saying that even if the investment does not look very promising, when the whole distribution is looked at, the management can draw the further conclusion that if it is possible to fulfill the localization requirement, keep high average capacity factor, and make sure that the CapEx level is not higher than $120 \%$ of the limit, then the investment is likely to be profitable. This information gives actionable management information that the management can start analyzing - the question "are the circumstances that lead to profitability that have thus been uncovered within the reach of the organization or not and if they are, then what are the operational steps to reach them?".

\section{DISCUSSION AND CONCLUSIONS}

Simulation-based techniques are commonly used in many fields for the analysis of the joint effects of uncertain variables. One of the fields where simulation techniques have been found useful is investment analysis, where simulations are run based on profitability models built for the studied investments. Typically simulation is used as a tool to create distributions of possible outcomes that are presented in the form of histograms - in this paper we posit that when complex problems, with multiple uncertain and simulated variables, are analyzed it makes sense to decompose the resulting distributions to find out more about the results. For this purpose we present a new method that we have called "simulation decomposition".

This idea of the method is shortly to create sub-distributions of the overall simulation, by categorizing states of the uncertain variables simulated and by way of forming combinations of different variables' states and collecting the simulated outputs from each scenario. This way the simulated information is not only used to create a single "overall" distribution from results, but also a number of sub-distributions for the scenarios that the state combinations of the uncertain variables represent. The information that is revealed increases the value of the simulation, because by better understanding the connection between the circumstances (variable states) and the results to which they have lead (simulation results) decision-makers can make better use of the analysis results. 
In fact, if the thresholds separating the states of the uncertain variables are such that they represent different levels of effort, or actions such as investments, the information can be formed into actionable "march orders", for example, in the context of the numerical illustration presented in the paper. The ability to trace the influence of different states of the uncertain factors on the results (project profitability), and the possibility to compare the outcomes of different scenarios and probabilities of different scenarios offer, in our view, enhances decision-support that can be given to managers with simulation.

Using smart graphical presentation and the types of tables and separate scenario distributions will enhance the intuitive understandability of the obtained results. On top of the described benefits the proposed method is rather easy to implement and does not necessarily require any changes to the models that underlie the simulation procedure. The method is also generally usable and is not context dependent.

The numerical case illustration based on a solar PV power plant investment analysis reveals the added analytical insights brought about by the simulation decomposition that can be used to create action points for management of such investments. The method can be used in similar investment analyses, even without such natural thresholds between states of variables that were found in the example used, in such cases the thresholds need to be managerially estimated and decided. Furthermore, the insights uncovered in the numerical case illustration also contribute to the study of the Russian renewable energy policy.

In a broader management decision-support context, the simulation decomposition approach outperforms classical techniques to analyzing sensitivity of the results. It possesses all the features of the traditional simulation and provides more insights on top of the classical simulation practice. The proposed approach reflects some features of sensitivity and scenario analysis that are typically used to complement investment analyses and can also be understood as a more developed approach in the same vein with these methods.

Further research into the topic of distribution decomposition will include studying the real world situation, where managers are asked to identify the thresholds between the different states of the required variables and extending this thinking into investment analysis within the possibilistic framework. 


\section{ACKNOWLEDGMENT}

The authors would like to acknowledge the financial support received by M. Kozlova from Fortum Foundation.

The financing has not included any involvement by the sponsor in the research process.

\section{REFERENCES}

1. RYAN, P. A., RYAN, G. P. (2002). "Capital budgeting practices of the fortune 1000: How have things changed". Journal of Business and Management, Vol. 8, No. 4, p. 355-364.

2. GRAHAM, J. R., HARVEY, C. R. (2001). "The theory and practice of corporate finance: Evidence from the field". Journal of Financial Economics, Vol. 60, No. 2-3, p. 187-243.

3. BLOCK, S. (2007). "Are "real options" actually used in the real world?". The Engineering Economist, Vol. 52, No. 3, p. 255-267.

4. TRIGEORGIS, L. (1995). Real options: An overview. Praeger Westport, CT.

5. AMRAM, M., KULATILAKA, N. (1998). Real options: Managing strategic investment in an uncertain world. Oxford University Press.

6. BoYLE, P. P. (1977). "Options: A Monte Carlo approach". Journal of Financial Economics, Vol. 4, No. 3, p. 323-338.

7. SHEEL, A. (1995). "Monte Carlo simulations and scenario analysis - decision-making tools for hoteliers". The Cornell Hotel and Restaurant Administration Quarterly, Vol. 36, No. 5, p. 18-26.

8. JOHNSON, S., TAYLOR, T., FORD, D. (2006). Using system dynamics to extend real options use: Insights from the oil \& gas industry. International System Dynamics Conference, pp. 23-27.

9. O'REGAN, B., MOLES, R. (2001). "A system dynamics model of mining industry investment decisions within the context of environmental policy". Journal of Environmental Planning and Management, Vol. 44, No. 2, p. 245-262.

10. O'Regan, B., Moles, R. (2006). "Using system dynamics to model the interaction between environmental and economic factors in the mining industry". Journal of Cleaner Production, Vol. 14, No. 8, p. 689-707.

11. Sontamino, P., Drebenstedt, C. (2014). A prototype decision making tool of coal mine planning using system dynamics model. Mine planning and equipment selection (pp. 1475-1484) Springer.

12. TAN, B., Anderson, E. G., Dyer, J. S., PARKER, G. G. (2010). "Evaluating system dynamics models of risky projects using decision trees: Alternative energy projects as an illustrative example". System Dynamics Review, Vol. 26, No. 1, p. 1-17.

13. VITHAYASRICHAREON, P., MACGILL, I. F. (2012). "A Monte Carlo based decision-support tool for assessing generation portfolios in future carbon constrained electricity industries". Energy Policy, Vol. 41, p. 374-392.

14. MonjAS-BARROSO, M., BALIBREA-INIESTA, J. (2013). "Valuation of projects for power generation with renewable energy: A comparative study based on real regulatory options". Energy Policy, Vol. 55, No. 0, p. 335-352.

15. ReUter, W. H., FusS, S., SzolgayovÁ, J., OBersteINER, M. (2012). "Investment in wind power and pumped storage in a real options model". Renewable and Sustainable Energy Reviews, Vol. 16, No. 4, p. 2242-2248. 
16. BAStIAN-PINTO, C., BRANDÃO, L., AlVES, M. D. L. (2010). "Valuing the switching flexibility of the ethanol-gas flex fuel car". Annals of Operations Research, Vol. 176, No. 1, p. 333-348.

17. BOOMSMA, T. K., MEADE, N., FLETEN, S. (2012). "Renewable energy investments under different support schemes: A real options approach". European Journal of Operational Research, Vol. 220, No. 1, p. 225-237.

18. YANG, M., NGuYen, F., De T'Serclaes, P., BuChNeR, B. (2010). "Wind farm investment risks under uncertain CDM benefit in China". Energy Policy, Vol. 38, No. 3, p. 1436-1447.

19. KOZLOVA, M., COLLAN, M., LUUKKA, P. (2015). Comparing Datar-Mathews and fuzzy pay-off approaches to real option valuation. Real Option Workshop, Lappeenranta, Finland. pp. 29-34.

20. MATHEWS, S., DATAR, V., JOHNSON, B. (2007). "A practical method for valuing real options: The Boeing approach". Journal of Applied Corporate Finance, Vol. 19, No. 2, p. 95-104.

21. DATAR, V. T., AND MATHEWS, S. H. (2004). "European real options: An intuitive algorithm for the Black-Scholes formula". Journal of Applied Finance, Vol. 14, No. 1, p. 45-51.

22. JAIMUNGAL, S., LAWRYSHYN, Y. (2015). Incorporating managerial information into real option valuation. Commodities, energy and environmental finance (pp. 213-238) Springer.

23. Rane, S. U., Shet, T., Sridhar, E., Bhele, S., Gaikwad, V., Agale, S., et Al. (2014). "Interobserver variation is a significant limitation in the diagnosis of burkitt lymphoma". Indian Journal of Medical and Paediatric Oncology: Official Journal of Indian Society of Medical \& Paediatric Oncology, Vol. 35, No. 1, p. 44.

24. García-Redondo, F., LóPez-VAllejo, M., ITUERO, P., BARRio, C. L. (2012). A CAD framework for the characterization and use of memristor models. Synthesis, Modeling, Analysis and Simulation Methods and Applications to Circuit Design (SMACD), 2012 International Conference On, pp. 25-28.

25. SAVOLAINEN, J., COLLAN, M., LUUKKA, P. (2016). "Analyzing operational real options in metal mining investments with a system dynamic model". The Engineering Economist, No. just-accepted, p. 00-00.

26. RIDLEHOOVER, J. (2004). "Applying Monte Carlo simulation and risk analysis to the facility location problem". The Engineering Economist, Vol. 49, No. 3, p. 237-252.

27. NemBHARD, H. B., SHI, L., AKTAN, M. (2003). "A real options design for product outsourcing". The Engineering Economist, Vol. 48, No. 3, p. 199-217.

28. GovernMENT OF RuSSIAN FEDERATION. (2013a). 28 may 2013 Decree \#449 on the Mechanism of Promoting the use of Renewable Energy in the Wholesale Market of Electric Energy and Power.

29. GovernMment OF RusSian FEDERATION. (2013b). 28 may 2013 Resolution \#861-R on Amendments being made to Resolution \#1-R 8.01.2009 on the Main Directions for the State Policy to Improve the Energy Efficiency of the Electricity Sector on the Basis of Renewable Energy Sources for the Period Up to 2020.

30. BOUTE, A. (2012). "Promoting renewable energy through capacity markets: An analysis of the Russian support scheme". Energy Policy, Vol. 46, p. 68-77.

31. VASILEVA, E., VILJAINen, S., SULAMAA, P., KuleShOV, D. (2015). "RES support in Russia: Impact on capacity and electricity market prices". Renewable Energy, Vol. 76, No. 0, p. 82-90.

32. INTERNATIONAL FINANCE CORPORATION. (2011). Renewable energy policy in Russia: Waking the green giant. (Retrieved September

2015), available 
http://www.ifc.org/wps/wcm/connect/6239e00040c80fcdb865bd5d948a4a50/Green+Giant Eng.pdf?MOD=AJPER $\underline{E S}$

33. KOZLOVA, M. (2015) Analyzing the effects of the new renewable energy policy in Russia on investments into wind, solar and small hydro power. (Master's thesis, Lappeenranta University of Technology), p. 104.

34. MOONEY, C. Z. (1997). Monte Carlo simulation. Sage Publications.

35. KOZLOVA, M., COLLAN, M. (2016). "Modeling the effects of the new Russian capacity mechanism on renewable energy investments". Energy Policy, Vol. 95, p. 350-360. 
APPENDIX 1. Two-sample Kolmogorov-Smirnov test

Kolmogorov-Smirnov statistics represents the test decision for the null hypothesis that the data in vectors are from the same continuous distribution. The alternative hypothesis is that data vectors are from different continuous distributions. The result is 1 if the test rejects the null hypothesis at the $5 \%$ significance level, and 0 otherwise.

Table 1.1. Kolmogorov-Smirnov statistics

\begin{tabular}{|c|c|c|c|c|c|c|c|c|c|c|c|c|c|}
\hline & Sc. 1 & Sc. 2 & Sc. 3 & Sc. 4 & Sc. 5 & Sc. 6 & Sc. 7 & Sc. 8 & Sc. 9 & Sc. 10 & Sc. 11 & Sc. 12 & Total \\
\hline Sc. 1 & 0 & 1 & 1 & 1 & 1 & 1 & 1 & 1 & 1 & 1 & 1 & 1 & 1 \\
\hline Sc. 2 & & 0 & 1 & 1 & 1 & 1 & 1 & 1 & 1 & 1 & 1 & 1 & 1 \\
\hline Sc. 3 & & & 0 & 1 & 1 & 1 & 1 & 1 & 1 & 1 & 1 & 1 & 1 \\
\hline Sc. 4 & & & & 0 & 1 & 1 & 1 & 1 & 1 & 1 & 1 & 1 & 1 \\
\hline Sc. 5 & & & & & 0 & 1 & 1 & 1 & 1 & 1 & 1 & 1 & 1 \\
\hline Sc. 6 & & & & & & 0 & 1 & 1 & 1 & 1 & 1 & 1 & 1 \\
\hline Sc. 7 & & & & & & & 0 & 1 & 1 & 1 & 1 & 1 & 1 \\
\hline Sc. 8 & & & & & & & & 0 & 1 & 1 & 1 & 1 & 1 \\
\hline Sc. 9 & & & & & & & & & 0 & 1 & 1 & 1 & 1 \\
\hline Sc. 10 & & & & & & & & & & 0 & 1 & 1 & 1 \\
\hline Sc. 11 & & & & & & & & & & & 0 & 1 & 1 \\
\hline Sc. 12 & & & & & & & & & & & & 0 & 1 \\
\hline Total & & & & & & & & & & & & & 0 \\
\hline
\end{tabular}


Table 2.2. P-values

\begin{tabular}{|c|c|c|c|c|c|c|c|c|c|c|c|c|c|}
\hline & Sc. 1 & Sc. 2 & Sc. 3 & Sc. 4 & Sc. 5 & Sc. 6 & Sc. 7 & Sc. 8 & Sc. 9 & Sc. 10 & Sc. 11 & Sc. 12 & Total \\
\hline Sc. 1 & 1 & 0 & 0 & 0 & 0 & 0 & 0 & 0 & 0 & 0 & 0 & 0 & 0 \\
\hline Sc. 2 & & 1 & 0 & 0 & 0 & 0 & 0 & 0 & 0 & 0 & 0 & 0 & 0 \\
\hline Sc. 3 & & & 1 & 0 & 0 & 0 & 0 & 0 & 0 & 0 & 0 & 0 & 0 \\
\hline Sc. 4 & & & & 1 & 0 & 0 & 0 & 0 & 0 & 0 & 0 & 0 & 0 \\
\hline Sc. 5 & & & & & 1 & 0 & 0 & 0 & 0 & 0 & 0 & 0 & 0 \\
\hline Sc. 6 & & & & & & 1 & 0 & 0 & 0 & 0 & 0 & 0 & 0 \\
\hline Sc. 7 & & & & & & & 1 & 0 & 0 & 0 & 0 & 0 & 0 \\
\hline Sc. 8 & & & & & & & & 1 & $3,6 \mathrm{E}-154$ & 0 & 0 & 0 & 0 \\
\hline Sc. 9 & & & & & & & & & 1 & 0 & 4,2E-296 & 0 & 0 \\
\hline Sc. 10 & & & & & & & & & & 1 & 0 & 7,3E-128 & 0 \\
\hline Sc. 11 & & & & & & & & & & & 1 & 0 & 0 \\
\hline Sc. 12 & & & & & & & & & & & & 1 & 0 \\
\hline Total & & & & & & & & & & & & & 1 \\
\hline
\end{tabular}

